Research Journal of Applied Sciences 7 (6): 286-292, 2012

ISSN: $1815-932 \mathrm{X}$

(C) Medwell Journals, 2012

\title{
The Application of the Residue Number System in Digital Image Processing: Propose a Scheme of Filtering in Spatial Domain
}

\author{
${ }^{1}$ Shahram Moharrami and ${ }^{2}$ Davar Kheirandish Taleshmekaeil \\ ${ }^{1}$ Department of Computer Engineering, ${ }^{2}$ Young Researchers Club, \\ Parsabad Moghan Branch, Islamic Azad University, Parsabad Moghan, Iran
}

\begin{abstract}
This study tries to investigate, the application of the Residue Number System in Digital Image Processing and propose a scheme in digital image filtering of spatial domain based on the Residue Number System using the integration circuits with high speeds and security and low power for Digital Image Processing. In this method the selected mask in the residue system moves on to the desired image and then the gray levels of the pixels of new images were computed based on mask values. All computations of this method will be done in the Residue Number System. Then, the binary circuits were designed for residue conversion and vice versa in which Matlab Software and a VLSI tools were used for simulation. Preliminary simulation results showed that the proposed scheme has the ability of digital image filtering.
\end{abstract}

Key words: Digital image processing, Residue Number System (RNS), Chinese Remainder Theorem (CRT), Look Up Table (LUT), filtering, spatial domain

\section{INTRODUCTION}

Undoubtedly Digital Image Processing has a wide range of applications in different scientific fields. Such as machine vision, medical application, satellite pictography, military and security sciences, automatic controlling of products, agriculture applications, urbanism, graphical and multimedia arts. Usefulness of these applications most depends on simplicity of hardware implementation, low consumption power, high speed operation of processing circuits and high security level. So their applications are very important in circuits with Very Large Scale Integration (VLSI) technology (Wang et al., 2004).

Since, there are a large number of pixels in one digital image and also operation of Digital Images Processing requires many computation operators on available pixels, so researchers require techniques for decreasing consumption power and increasing processing speed in order to perform integrated circuits which can do the Digital Image Processing. One of the methods for designing circuits with high scale integration and speed and low consumption power is using the Residue Numbers System. One of the properties of RNS is distribution of limited carry digit between moduli in computations which performs computations simultaneously on residue numbers instead of large numbers at the same time. This property considerably increases the speed of computation and decrease consumption power. Another advantage of this system is its relative security for changing weighted numbers to residue numbers and vice versa in which we need a selective moduli set without which we can not use obtained values (Wang et al., 2003).

Residue Number System are mostly used in iterated operations of summation, subtraction and multiplying. Digital Image Processing is one of these applications that generally increases computation speed and decreases complexity of hardware circuits chip area and implementation of a good VLSI circuits.

\section{BACKGROUND}

Digital image filtering: One attractive section in Digital Image Processing is images enhancement. Application of this processing on digital images improves digitalized image presentation qualities and also it is used in many fields such as sharpening, denoising, edge detection, thresholding and filtering of digital images. In this study, researchers perform filtering of digital images in spatial domain of with gray levels. Then, researchers investigated the effects of available filters like Prewitt, Gaussian, Laplacian, Sobel and other filters on images processing (Gonzalez and Woods, 2002).

Researchers use a two dimensional array (matrix) $\mathrm{M} \times \mathrm{N}$ for representing one image. Values of each elements of this array show brightness severity of image on that point and each element has 8 bit value that may vary between $0-255$. This image is defined as following in matlab:

Corresponding Author: Davar Kheirandish Taleshmekaeil, Department of Computer Engineering, Parsabad Moghan Branch, Islamic Azad University, Parsabad Moghan, Iran 


$$
\mathrm{F}(\mathrm{x}, \mathrm{y})=\left[\begin{array}{cccc}
\mathrm{F}(1,1) & \mathrm{F}(1,2) & \ldots & \mathrm{F}(1, \mathrm{~N}) \\
\mathrm{F}(2,1) & \mathrm{F}(2,2) & \ldots & \mathrm{F}(2, \mathrm{~N}) \\
\cdot & . & . & . \\
\mathrm{F}(\mathrm{M}, 1) & \mathrm{F}(\mathrm{M}, 2) & \ldots & \mathrm{F}(\mathrm{M}, \mathrm{N})
\end{array}\right]
$$

Combination of function and mask for changing gray levels is called filter. Researchers can perform a linear filter by multiplying all factors in defined mask factors as an adjacent element and sum up all obtained values (Gonzales and Woods, 2002).

If we have on image with gray levels and also if we want to perform filtering operation in spatial domain then we should move suggested mask on the image and use it adjacent to pixel values of image so, we would have a new image with gray levels calculated according to suggested mask. By multiplying mask values in factors adjacent to target pixel we can obtain the pixel value in the new image.

$$
g(x, y)=\sum_{s=-a}^{a} \sum_{t=-b}^{b} w(s, t) f(x+s, y+t)
$$

Where:

$\mathrm{a}$ and $\mathrm{b}=$ The positive integers

$\mathrm{f}(\mathrm{x}, \mathrm{y})=$ A function for input image

$\mathrm{w}(\mathrm{s}, \mathrm{t})=\mathrm{A}$ function for given filter

$\mathrm{g}(\mathrm{x}, \mathrm{y})=\mathrm{A}$ function for values of output image

For example, researchers can consider mask factors in the form of $3 \times 3$ as following:

\begin{tabular}{|l|l|l|}
\hline $\mathrm{w}(-1,1)$ & $\mathrm{w}(-1,0)$ & $\mathrm{w}(-1,-1)$ \\
\hline $\mathrm{w}(0,1)$ & $\mathrm{w}(0,0)$ & $\mathrm{w}(0,-1)$ \\
\hline $\mathrm{w}(1,1)$ & $\mathrm{w}(1,0)$ & $\mathrm{w}(1,-1)$ \\
\hline
\end{tabular}

and other correspondent pixels have values as following:

\begin{tabular}{|l|l|l|}
\hline$f(x-1, y+1)$ & $f(x-1, y)$ & $f(x-1, y-1)$ \\
\hline$f(x, y+1)$ & $f(x, y)$ & $f(x, y-1)$ \\
\hline$f(x+1, y+1)$ & $f(x+1, y)$ & $f(x+1, y-1)$ \\
\hline
\end{tabular}

now two correspondents matrixes are multiplied and finally we sum value:

$$
\sum_{s=-1}^{1} \sum_{t=-1}^{1} w(s, t) f(x+s, y+t)
$$

Therefore, generally there are 3 stages for filtering operation as follow:
- Location of mask on present mask

- Multiplication of filter factors in adjacent correspondent

- Total sum of obtained values (Gonzales and Woods, 2002)

When we apply filter for pixel values some parts of mask would be out of image. Reaserch have two methods for solving this problem. First, given image would be smaller than original image regardless of margins. Second, researchers can put series of zero numbers in margins. Different kinds of filters for more quality improvement of image are divided in to two categories.

Low-Pass filter which passes through low frequency pixels and masks changes on the high frequency pixels that leads to masking quiet images.

High-Pass filter which passes through high frequency pixels and makes changes on the low frequency pixels and leads to making images with edges and noises.

Some available filters which are used for different applications such as sharpening, edge detection and denoising etc are as following:

- Prewitt filters for edge detection:

$$
P_{x}=\left[\begin{array}{lll}
-1 & 0 & 1 \\
-1 & 0 & 1 \\
-1 & 0 & 1
\end{array}\right], P_{y}=\left[\begin{array}{ccc}
-1 & -1 & -1 \\
0 & 0 & 0 \\
1 & 1 & 1
\end{array}\right]
$$

- Sobel filters for edge detection:

$$
P_{x}=\left[\begin{array}{lll}
-1 & 0 & 1 \\
-2 & 0 & 1 \\
-1 & 0 & 1
\end{array}\right], P_{y}=\left[\begin{array}{ccc}
-1 & -2 & 1 \\
0 & 0 & 0 \\
1 & 2 & 1
\end{array}\right]
$$

Researchers want to perform this operation through Residue Number System.

Residue Number System: Residue Number System according to moduli set is defined as $\left(\mathrm{m}_{\mathrm{i}}\right)_{\mathrm{i}}=1,2, \ldots, \mathrm{n}$ which relatively prime numbers that is:

$$
\operatorname{GCD}(i, j)=1, i \neq j
$$

Where decimal value of $\mathrm{X}$ in non-weighted of Residue Number System is shown as following:

$$
\begin{gathered}
X=\left(x_{1}, x_{2}, \ldots, x_{n}\right) \\
x_{i}=X \bmod m_{i} \\
\exists q \Rightarrow X=q_{i} \cdot m_{i}+x_{i}
\end{gathered}
$$

where, $i=1,2,3, \ldots, N$. Where $x_{i}$ is given by residue of $X$ on modulo $\mathrm{m}_{\mathrm{i}}$ (Ammar et al., 2001). 


$$
\mathrm{M}=\prod_{\mathrm{i}=1}^{\mathrm{n}} \mathrm{m}_{\mathrm{i}}
$$

is dynamic limitation of this number in Residue Number System of $\mathrm{X}$ in this system which can be shown as $\mathrm{X}=\left(\mathrm{x}_{1}, \mathrm{x}_{2}, \ldots, \mathrm{x}_{\mathrm{n}}\right)$ which initially shown as $0 \leq \mathrm{x}_{\mathrm{i}}<\mathrm{m}_{\mathrm{i}}$. This value for each integer number of $\mathrm{X} \in[\alpha, \mathrm{M}+\alpha] \alpha$ is an integer number is unique (Freking and Parhi, 1997; Hiasat, 2002).

Arithmetic operation can be classified by two groups, i.e., simple and complex operations. Adding, subtracting and multiplying operations are among simple operation group. Divisions, sign detection and magnitude comparison are among complex operation group (Konstantinides and Bhaskaran, 1992; Soderstrand et al., 1986). Addition and subtraction in system of residue numbers are:

$$
\begin{aligned}
& X=\left(x_{1}, x_{2}, \ldots, x_{n}\right) \\
& Y=\left(y_{1}, y_{2}, \ldots, y_{n}\right) \\
& |X \pm Y|_{M}=\left(\left|x_{1} \pm y_{1}\right|_{m_{1}},\left|x_{2} \pm y_{2}\right|_{m_{2}}, \ldots,\left|x_{n} \pm y_{n}\right|_{m_{n}}\right)
\end{aligned}
$$

and also, multiplying operation in Residue Number Systems includes:

$$
\begin{aligned}
& X=\left(x_{1}, x_{2}, \ldots, x_{n}\right) \\
& Y=\left(y_{1}, y_{2}, \ldots, y_{n}\right) \\
& |X . Y|_{M}=\left(\left|x_{1} \cdot y_{1}\right|_{m_{1}},\left|x_{2} \cdot y_{2}\right|_{m_{2}}, \ldots,\left|x_{n} \cdot y_{n}\right|_{m_{n}}\right)
\end{aligned}
$$

Researchers also, used Chinese Remainder Theorem in conversion Residue Number System in to weighted system (Wang et al., 2003):

$$
\begin{aligned}
& \mathrm{X}=\left\langle\sum_{\mathrm{i}=1}^{\mathrm{n}}\left(\mathrm{x}_{\mathrm{i}} \mathrm{N}_{\mathrm{i}}\right)_{\mathrm{m}_{\mathrm{i}}} \times \mathrm{M}_{\mathrm{i}}\right\rangle_{\mathrm{M}}, \\
& \mathrm{M}=\prod_{\mathrm{i}=1}^{\mathrm{n}} \mathrm{m}_{\mathrm{i}}, \quad \mathrm{M}_{\mathrm{i}}=\frac{\mathrm{M}}{\mathrm{m}_{\mathrm{i}}}, \\
& \mathrm{N}_{\mathrm{i}}=\left\langle\mathrm{M}_{\mathrm{i}}^{-1}\right\rangle_{\mathrm{m}_{\mathrm{i}}}, \quad \mathrm{i}=1,2, \ldots, \mathrm{n}
\end{aligned}
$$

\section{PROPOSED SCHEME}

Proposed scheme for spatial domain: According to filtering of digital images we just have used algebraic operations of multiplication and summation on value of digitalized images. So, filtering operations related to spatial domain by using Residue Numbers System can be performed. The reason of using this system is based on increasingly improvement of available pixel values in digital image according to technological improvements of images. Therefore, filtering operation of digital needs many multiplication and summation of available pixel values with correspondent mask. Hereby we can conclude that implementation of filtering operation or generally operations related to the Digital Images Processing need a large number of overloads for processing speed and consumption power. So, we should look for solutions in order to increase the speed of operation which are mostly multiplication and summation of pixel values of images. Considering the point that values of image factors (pixels) in a limited area for example in images with gray levels between 0-255, researchers use Residue Numbers System that one of their properties is that they have a high speed of operation in some limited numbers. Residue Numbers System is used mostly in applications of some limited numbers. Summation and subtraction is repeated in these operations. Many of these operations are dependent on having high speed processing, simplification of hardware implementation, low consumption power and increased security.

So, their application in technology of circuits with Very Large Scale Integrations (VLSI) is very important for Digital Image Processing. Architectures of Residue Number System can be used by a combinative circuits, LUT inter memories or combination of them. Since, Residue Numbers System has no continual carry so it performs operations according to a limited presentation and a look-up table with high speed. Advantage of high speed in Residue Number System in parallel highlights its usefulness for low consumption power of digital image filtering.

Therefore by using of Chinese Remainder Theorem and look up table and some limitations on numbers (relative binary modules and dynamic limitation), researchers can perform operations on numbers through different methods without missing their information. Regarding the pixel values in digital images between $0-255$ it is easy to do this operation with this system.

Researchers can use modified Chinese Remainder Theorem in changing residue system to weighted numbers system since simple CRT and dynamic limitation of $\mathrm{M}$ For large values have less efficiency for implementation. So we can use modified CRT with a set of reduced modules which reduces dynamic limitation:

$$
\mathrm{M}=\prod_{\mathrm{i}=1}^{\mathrm{m}} \mathrm{m}_{\mathrm{i}}
$$

of simple CRT to:

$$
\mathrm{M}=\prod_{\mathrm{i}=2}^{\mathrm{m}} \mathrm{m}_{\mathrm{i}}
$$


which leads to increase speed and efficacy and decrease consumption function:

$$
\mathrm{X}=\mathrm{x}_{1}+\mathrm{p}_{1}\left|\sum_{\mathrm{i}=1}^{\mathrm{m}} \mathrm{w}_{\mathrm{i}} \mathrm{x}_{\mathrm{i}}^{\prime}\right|_{\mathrm{p}_{2} \ldots \mathrm{p}_{\mathrm{m}}}
$$

where, $\mathrm{m}>1$;

$$
\mathrm{w}_{1}=\frac{\mathrm{N}_{1}\left|\mathrm{~N}_{1}^{-1}\right|_{\mathrm{p}_{1}^{-1}}}{\mathrm{p}_{1}}
$$

$\mathrm{w}_{\mathrm{i}}=\mathrm{N}_{\mathrm{i}} / \mathrm{p}_{1}$, for $\mathrm{i}=2,3, \ldots, \mathrm{m}, \mathrm{x}_{1}{ }^{\prime}=\mathrm{x}_{1}$ and:

$$
\mathrm{x}_{\mathrm{i}}^{\prime}=\left|\mathrm{N}_{\mathrm{i}}^{-1} \mathrm{x}_{\mathrm{i}}\right|_{\mathrm{p}_{\mathrm{i}}}, \mathrm{i}=2,3, \ldots, \mathrm{m}
$$

Transfer circuits design $(B / R$ and $R / B)$ : $I n$ order to process operations by Residue Number System, it is necessary to have transform binary number system to Residue Number System. So, we should design circuits initially to receive pixel values of images and change them to Residue Number System and then perform Digital Images Processing (filtering) that should be done on residue numbers. Then, new values of pixels in the form of residue numbers should be changed to binary numbers. So we classify this section in to 3 main parts:

- Transforming of pixel values of weighted system to residue number system (transformer $\mathrm{B} / \mathrm{R}$ )

- Performing processing operation of images with high speed in limited dynamic of Residue Number System

- Transforming pixel values of residue number system to previous weighted system (transformer $\mathrm{R} / \mathrm{B})$
Part 1: Transforming of pixel values of weighted system to Residue Number System, includes following steps:

- Reading the pixel values of the main digital image as decimal or binary numbers

- Choosing appropriate module for Residue Number System which covers limitation of digital image pixels

- Making Look Up Table (LUT) for faster processing

- Taking pixel values of the main digital image to RNS with selected module in the second stage

- Block diagram of $\mathrm{B} / \mathrm{R}$ transformer is shown in Fig. 1

Part 2: Image processing operation of filtering, performs in the Residue Number System.

Part 3: Transforming pixel values of Residue Number System to previous weighted system includes following stages:

- Receiving pixel values in the form of Residue Number System

- Identification of residue numbers

- Transforming of each residue number to its original form by using Look Up Table (LUT)

Block diagram of R/B is shown in Fig. 2.

Look Up Table (LUT): Look up table actually plays an important role in transforming process from Residue Number System to system of weighted numbers with high speed. This transformation can be done through two methods. One method by Boolean function and the other one by look up table. The second method is faster than the first one and is too also it useful for filtering of digital images. Look up table is made during transforming from weighted numbers to residue system

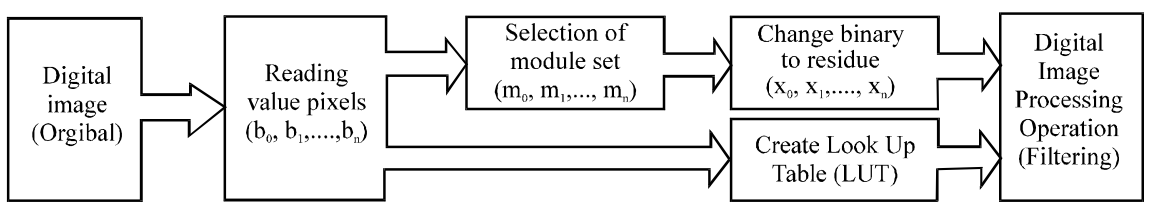

Fig. 1: Proposed scheme for transformer $\mathrm{B} / \mathrm{R}$ (Transforming from binary numbers to residue number)

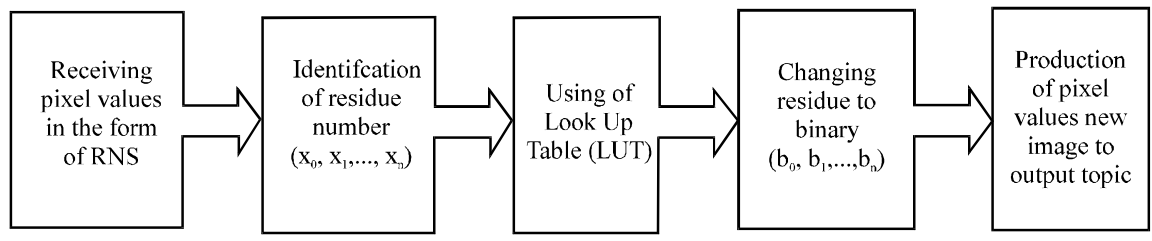

Fig. 2: Suggested design for transformer R/B (Transforming from system of residue numbers to binary numbers) 
just for available pixel values in produced digital images and it is used during transforming from new values of digital pixels to system of weighted numbers that naturally leads to faster transformation of values.

Selection of module set: Another issue in discussion about design and implementation of circuits transforming $\mathrm{R} / \mathrm{B}$ and $\mathrm{B} / \mathrm{R}$ might be about appropriate selection of module. There is a large number of choices available for RNS module set. The importance of using module is deals with dynamic limitation increasing processing speed and good implementation of VLSI.

Considering values of image factors (pixels) with gray levels in limited area between $0-255$, so researchers use module set for using Residue Number System which can cover these digital image pixels and also have relatively high security and have been chosen in order to transform pixel values in the selected module. Therefore, the most appropriate form of selected module set about operation of processing digital images can be $\left\{2^{\mathrm{n}-1}+1,2^{\mathrm{n}}-1,2^{\mathrm{n}}\right\}$ in which by substituting $\mathrm{n}=3$, researchers would have $\{5,7,8\}$. This set compromises a dynamic limited module of $\mathrm{M}=5 \times 7 \times 8=280$ is $0, \mathrm{M}$ and have the ability of covering illustrative limited pixel values of digital images.

\section{SECURITY}

Considering this fact that values of out put transformer B/R is in the form of Residue Number System and these values in Residue Numbers System are like a coded group of bites arranged in a specific order and their operations are cryptic so an unauthorized hearer unaware of the order of these parallel outputs and selected module can not use these values. This coded bite group without a selected set of modules by transformer $\mathrm{B} / \mathrm{R}$ can not be decoded and just $\mathrm{R} / \mathrm{B}$ transformer can transform these coded bite values to applicable values instead of pixels of images for a new image.

Therefore for using Residue Numbers System in Digital Images Processing, it is just enough to choose one specific module in order to provide security in all low and medium levels and there is no need to additional number of bits used for pixel values of images.

\section{SIMULATION AND COMPARISON}

Simulation: Suppose that digital image in processing has an image with gray levels. Researchers choose apart of image and perform processing on it. For example, pixel values of image are as following:

\begin{tabular}{|l|l|l|l|}
\hline 149 & 148 & 152 & 150 \\
\hline 150 & 151 & 152 & 147 \\
\hline 151 & 149 & 148 & 152 \\
\hline 148 & 150 & 149 & 151 \\
\hline
\end{tabular}

Mask values for moving on four middle values of image pixels are given as following which is known as one of the sharpening filters of Laplas.

\begin{tabular}{|c|c|c|}
\hline-1 & -1 & -1 \\
\hline-1 & 9 & -1 \\
\hline-1 & -1 & -1 \\
\hline
\end{tabular}

First, researchers should transform all available pixel values and mask values to residue numbers. To do so we need to choose a module set for Residue Number System. Recommended module for filtering of this set is $\left\{2 \mathrm{n}^{-1}+1,2^{\mathrm{n}}-1,2^{\mathrm{n}}\right\}$ in which we have a dynamic limitation equality $\mathrm{M}=5 \times 7 \times 8=280$ which is appropriate for digital images with gray levels. For selected pixel values we have:

\begin{tabular}{|c|c|c|c|}
\hline$\{4,2,5\}$ & $\{3,1,4\}$ & $\{2,5,0\}$ & $\{0,3,6\}$ \\
\hline$\{0,3,6\}$ & $\{1,4,7\}$ & $\{2,5,0\}$ & $\{2,0,3\}$ \\
\hline$\{1,4,7\}$ & $\{4,2,5\}$ & $\{3,4,4\}$ & $\{2,5,0\}$ \\
\hline$\{3,1,4\}$ & $\{0,3,6\}$ & $\{4,5,5\}$ & $\{1,4,7\}$ \\
\hline
\end{tabular}

For mask values researchers have:

\begin{tabular}{|l|l|l|}
\hline$\{4,6,7\}$ & $\{4,6,7\}$ & $\{4,6,7\}$ \\
\hline$\{4,6,7\}$ & $\{4,2,1\}$ & $\{4,6,7\}$ \\
\hline$\{4,6,7\}$ & $\{4,6,7\}$ & $\{4,6,7\}$ \\
\hline
\end{tabular}

Considering the use of look up table in inverse transforming from Residue Numbers System to weighted numbers system, leading to incensement of processing speed so, it is necessary to make look-up table for pixel values of digital images. Now researchers perform filtering operation on pixel values in the form of residue (Table 1). Obtained values in four middle points of given images are as following:

\begin{tabular}{|c|c|}
\hline$\{0,6,0\}$ & $\{1,3,3\}$ \\
\hline$\{2,2,6\}$ & $\{1,5,3\}$ \\
\hline
\end{tabular}


According to Chinese Remainder Theorem (CRT) and look up table we can transform values from Residue Number System to weighted number system:

\begin{tabular}{|c|c|}
\hline 160 & 171 \\
\hline 142 & 131 \\
\hline
\end{tabular}

Software considered for simulation is Matlab. First, researchers would receive mask values transformed in filtering to residue operation on pixel values of digital

Table 1: Value presentation of available pixels and its residue form

\begin{tabular}{lc}
$\begin{array}{l}\text { Value pixels for residue number } \\
\text { in module set }\{5,7,8\}\end{array}$ & $\begin{array}{c}\text { Value pixels in weighted } \\
\text { number system (Decimal) }\end{array}$ \\
\hline$\{2,0,3\}$ & 147 \\
$\{3,1,4\}$ & 148 \\
$\{4,2,5\}$ & 149 \\
$\{0,3,6\}$ & 150 \\
$\{1,4,7\}$ & 151 \\
$\{2,5,0\}$ & 152 \\
\hline
\end{tabular}

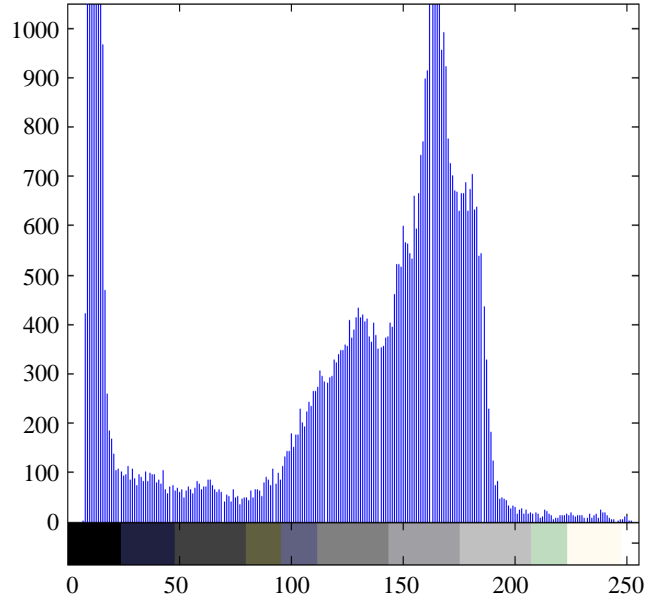

images in residue system. Results are shown as outputs with their histograms for input and output image obtained from transformer. Digital image input for image processing and its histogram are shown in Fig. 3.

After performing of processing, program output for given image and values of given mask and its histogram presented in Fig. 4.

Comparison: According to a large number of multiplication and summation in filtering of digital images, most of comparisons based on performance time of computations are available for filtering of digital images.

In this study, researchers describe some functions for filtering of digital images based on direct method and proposed method of Residue Number System in Matlab Software and give performing time in both methods in seconds based on available filter arrays of this software, then compute them and present them as diagram. Figure 5 shows this comparison in more details.

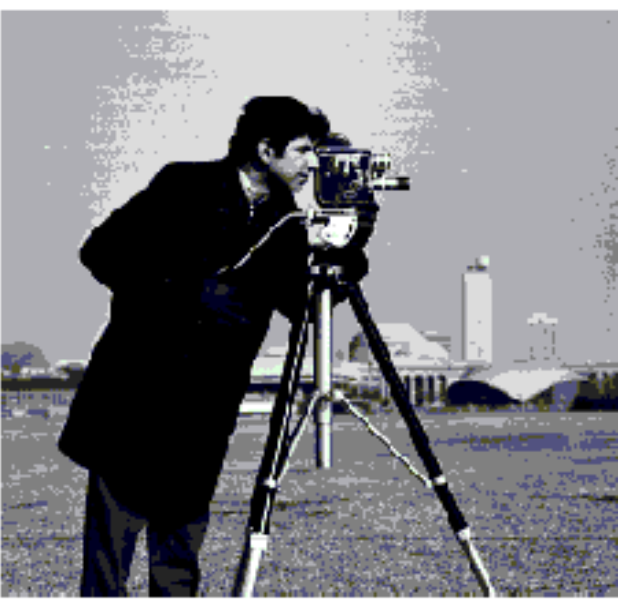

Fig. 3: Main image Cameraman.tif and its histogram (input B/R transformer)
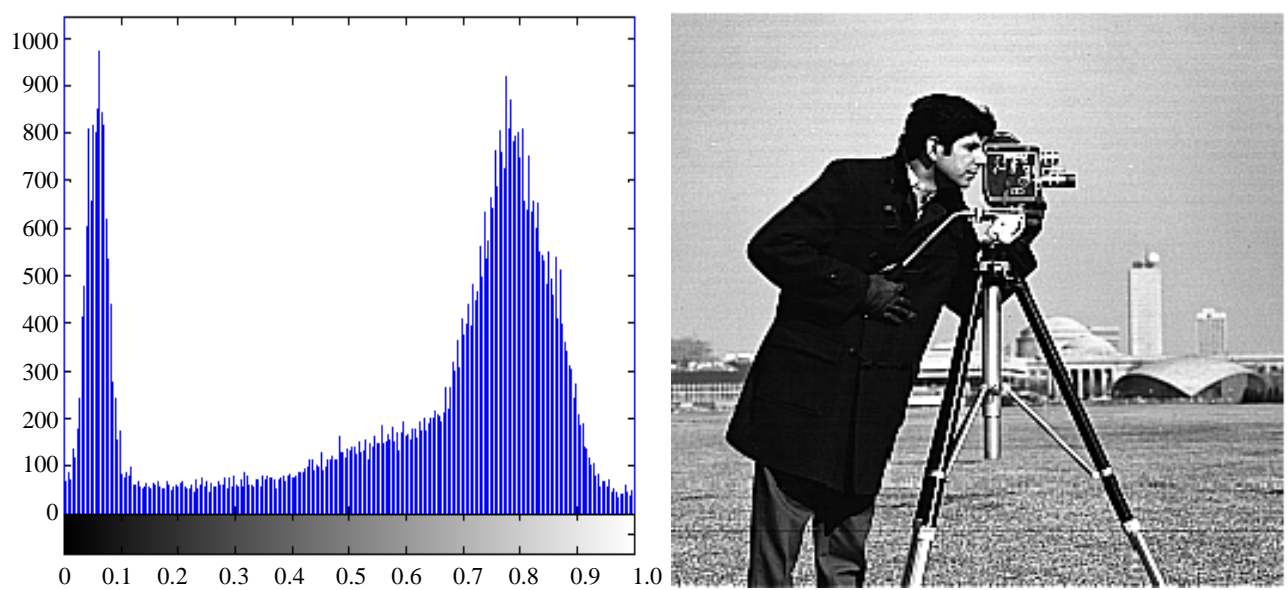

Fig. 4: Output R/B transformer and its histogram after filtering (clear representation of image) 


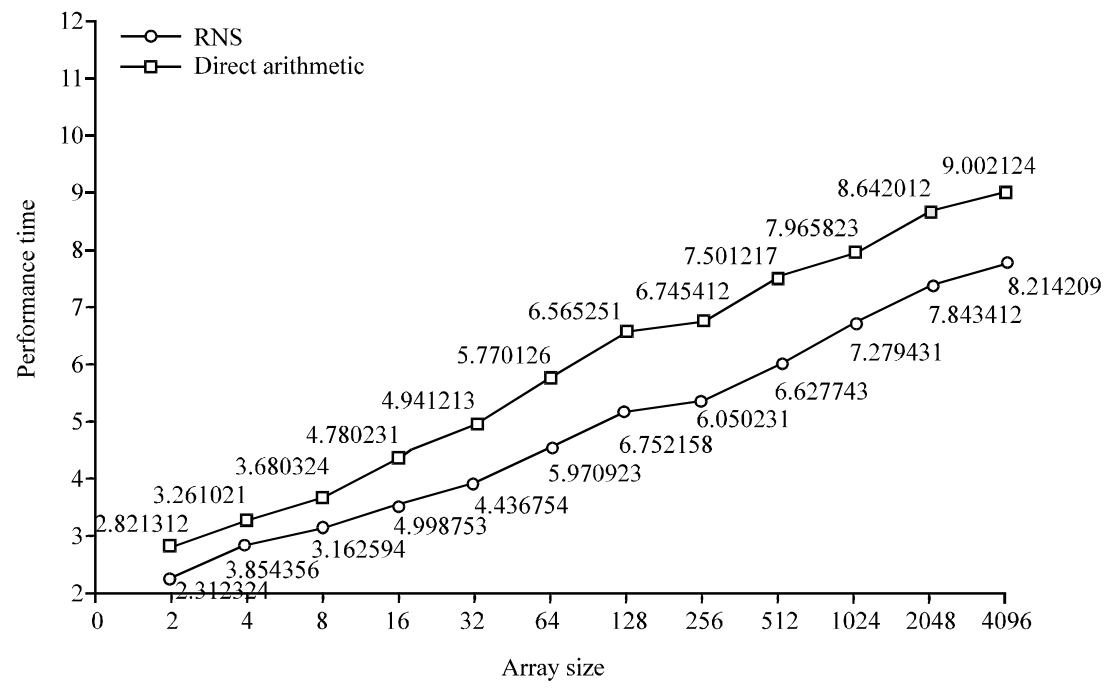

Fig. 5: Time performance diagram in seconds based on values of filter arrays

\section{CONCLUSION}

Processing operation of the image according to performing on pixel values of images and also number of pixels play a critical role in images with high quality. Therefore, each processing operation needs many algebric computations. In this study researchers worked on RNS application in Digital Image Processing and proposed a scheme of $\mathrm{B} / \mathrm{R}$ and $\mathrm{R} / \mathrm{B}$ transformers which leads to performance of VLSI with high speed and low consumption in Digital Images Processing. Implementation of filtering digital images in Residue Number System and selection of a good module and transforming process from system of residue with Chinese Remainder Theorem (CRT) and look up table caused speed incensement of operation, decreasing of consumption power, facilitating designed hardware and finally decreasing chip production. Results from Matlab simulation showed the ability of suggested design for filtering of given images.

\section{REFERENCES}

Ammar, A., A. Al Kabbany, M. Youssef and A. Amam, 2001. A secure image coding scheme using residue number system. Proc. National Radio Sci. Conf., 2: 399-405.
Freking, W.L. and K.K. Parhi, 1997. Low-power FIR digital filters using residue arithmetic. Proceedings of 31st Asilomar Conference on Signals, Systems and Computers, Nov. 2-5, Pacific Grove, CA, USA., pp: 739-743.

Gonzales, R.C. and R.E. Woods, 2002. Digital Image Processing. 2nd Edn., Prentice-Hall, New Jersey, USA., ISBN-10: 0201180758.

Hiasat, A.A., 2002. High-speed and reduced-area modular adder structures for RNS. IEEE Trans. Comput., 51: 84-89.

Konstantinides, K. and V. Bhaskaran, 1992. Monolithic architectures for image processing and compression. IEEE Comput. Graphics Appl., 12: 75-86.

Soderstrand, M.A., W.K. Jenkins, G.A. Jullien and F.J. Taylor, 1986. Residue Number System Arithmetic: Modern Applications in Digital Signal Processing. IEEE Press, New York, NY, USA.

Wang, W., M.N.S. Swamy and M.O. Ahmad, 2004. RNS application for digital image processing. Proceedings of the 4th IEEE International Workshop on System-on-Chip for Real-time Application, Jul. 19-21, Canada, pp: 77-80.

Wang, W., M.N.S. Swamy, M.O. Ahmad and Y. Wang, 2003. A study of the residue-to-binary converters for the three-moduli sets. IEEE Trans. Circuits Syst. I: Fundamental Theory Appl., 50: 235-243. 